\title{
Effects of plasma on gravitational lensing
}

\author{
Xinzhong $\mathrm{Er}^{1 \star}$, Shude Mao ${ }^{1,2}$ \\ ${ }^{1}$ National Astronomical Observatories, Chinese Academy of Sciences, 20 A Datun Road, Beijing 100012, China \\ 2 Jodrell Bank Centre for Astrophysics, University of Manchester, Alan Turing Building, Manchester M13 9PL, UK
}

Accepted -; received —; in original form 24 July 2018

\begin{abstract}
We study gravitational lensing when plasma surrounds the lens. An extra deflection angle is induced by the plasma in addition to the deflection generated by gravity. An inhomogeneous plasma distribution generates a greater effect than a homogeneous one, and may cause significant effects to be detected in low frequency radio observations (a few hundred $\mathrm{MHz}$ ). In particular, the lensed image positions will be different between optical and radio observations. The change of position due to a plasma can reach a few tens of milli-arcsec, which is readily detectable. One can use the position difference in different frequencies to estimate the density of plasma in the lens. The magnification ratios between multiple images are mainly determined by other properties of the lens, and are only weakly affected by the plasma. More importantly, we find that the strong lensing time delay will be affected by the plasma. Estimation of the Hubble constant from the time delay in low radio frequency observation may be slightly biased due to plasma in the lens. Unfortunately, the ionosphere of the Earth strongly affects low frequency radio observations. Thus our ability to detect the effect depends on how well we are able to calibrate out the ionosphere.
\end{abstract}

Key words: cosmology - gravitational lensing - Hubble constant - plasma

\section{INTRODUCTION}

The theory of gravitational lensing is well developed for light propagation in a vacuum. Deflection angles in a vacuum do not depend on the photon frequency but only on , the mass distribution of the lens. Lensing provides a way to study mass distribution in the universe, and is considered to be one of the most powerful tools in cosmology (see Bartelmann \& Schneider 2001; Treu 2010 for a review). In the study of cosmological gravitational lensing, several aspects have been investigated, for instance, the power spectrum of cosmic shear (e.g. Fu et al. 2008), the lensing probability of the separation of multiple images (e.g. Keeton \& Madau 2001; Li et al. 2007), the substructure of dark matter haloes (e.g. Li et al. 2013) and time delay between multiple images of strong lens systems (e.g. York et al. 2005; Paraficz \& Hiorth 2010; Suvu et al. 2013).

It is of interest to study gravitational lensing in a plasma since most lenses are surrounded by the interstellar or intergalatic medium. The properties of medium can be characterized by a refractive index $n$, through which an extra deflection angle can be introduced into the framework of vacuum lensing theory (see Bisnovatvi-Kogan \& Tsupko 2009, 2010; Tsupko \& Bisnovatyi-Kogan 2012, 2013, for more de- tail). Moreover, the plasma surrounding the lens is dispersive (the refractive index depends on the photon frequency) and inhomogeneous. Thus the extra deflection angle depends on the photon wavelength and the source position.

In general, the extra deflection angle due to plasma is several orders of magnitude smaller than the gravitational deflection. Only in the case of a high density plasma and low radio frequency observations (e.g. a few hundred $\mathrm{MHz}$ ), is it possible to detect the plasma lensing effects. Radio images are unaffected by extinction or microlensing. Thus radio lensing is well developed for studying substructures, and time delays (e.g. Koopmans et al. 2003; MacLeod et al. 2012). With new radio telescopes, e.g. LOFAR ${ }^{1}$ and SKA ${ }^{2}$, high sensitivity, and high spatial resolution will enable us to measure the weak lensing shear effect (Patel et al. 2013). Therefore, the effects due to plasma in radio lensing may be important in the future. On the other hand, gravitational lensing preserves the polarization properties of lensed sources, i.e. fraction and direction. Radiation of AGNs is polarized (e.g. Sluse et al. 2005). In fact, the polarization of some lensed sources has been already measured (Patnaik et al. 2001), and can be used to estimate the magnetic field of the lens using Faraday rotation (Narasimha \& Chitre 2008; Morozova et al. 2013). 
In this paper, we will focus on the effects due to plasma in gravitational lensing. Two simple models of plasma distribution are employed and two approximations of the plasma lensing equation are obtained and used. We discuss the effects in estimating weak lensing shear due to plasma in section 3. The effects on image positions and time delays are given in section 4. The cosmology we adopt in this paper is a $\Lambda$ CDM model with parameters based on the results from the PLANCK data (Planck Collaboration et al. 2013): $\Omega_{\Lambda}=0.6825, \Omega_{\mathrm{m}}=0.3175$, a Hubble constant $H_{0}=100 h$ $\mathrm{km} \mathrm{s}^{-1} \mathrm{Mpc}^{-1}$ and $h=0.671$.

\section{BASIC FORMALISM}

The fundamentals of gravitational lensing can be found in Bartelmann \& Schneider (2001). For its elegance and brevity, we shall use the complex notation. The thin-lens approximation is adopted, implying that the lensing mass distribution can be projected onto the lens plane perpendicular to the line-of-sight. We introduce angular coordinates $\theta$ with respect to the line-of-sight, and those on the source plane as $\boldsymbol{\beta}$. The lens equation can be written as

$$
\boldsymbol{\beta}=\boldsymbol{\theta}-\boldsymbol{\alpha}(\boldsymbol{\theta})
$$

where $\alpha$ is the deflection angle, and can be calculated from the lensing potential $\psi$

$$
\boldsymbol{\alpha}=\nabla \psi ; \quad \text { with } \quad \nabla=\frac{\partial}{\partial \theta_{1}}+\mathrm{i} \frac{\partial}{\partial \theta_{2}} .
$$

The lensing potential is determined by the dimensionless projected surface-mass density (lensing convergence),

$$
\begin{aligned}
\psi(\boldsymbol{\theta}) & =\frac{1}{\pi} \int_{\mathcal{R}^{2}} \mathrm{~d}^{2} \theta^{\prime} \kappa\left(\boldsymbol{\theta}^{\prime}\right) \ln \left|\boldsymbol{\theta}-\boldsymbol{\theta}^{\prime}\right| ; \\
\kappa(\boldsymbol{\theta}) & =\Sigma(\boldsymbol{\theta}) / \Sigma_{\mathrm{cr}}, \quad \text { where } \Sigma_{\mathrm{cr}}=\frac{c^{2}}{4 \pi G} \frac{D_{\mathrm{s}}}{D_{\mathrm{d}} D_{\mathrm{ds}}}
\end{aligned}
$$

is the critical surface mass density depending on the angulardiameter distances $D_{\mathrm{s}}, D_{\mathrm{d}}$ and $D_{\mathrm{ds}}$ from the observer to the source, the observer to the lens, and the lens to the source, respectively. $\Sigma(\boldsymbol{\theta})$ is the projected surface-mass density of the lens. To the lowest order, image distortions caused by gravitational lensing are described by the complex shear

$$
\gamma=\frac{1}{2}\left(\partial_{1}^{2} \psi-\partial_{2}^{2} \psi\right)+\mathrm{i} \partial_{1} \partial_{2} \psi
$$

which transforms a round source into an elliptical shape. The magnification for a point source is given by

$$
\mu=\frac{1}{(1-\kappa)^{2}-|\gamma|^{2}} .
$$

\subsection{Gravitational lensing in plasma}

In presence of a medium around the lens, the deflection angle will be slightly changed. In the weak field approximation, the lensing deflection angle in plasma is given by (Bisnovatyi-Kogan \& Tsupko 2009, 2010) as

$$
\begin{aligned}
\boldsymbol{\alpha}_{p l}=\boldsymbol{\alpha} & +\frac{\boldsymbol{\alpha}}{2}\left(\int_{0}^{\infty} \frac{1}{1-\omega_{e}^{2} / \omega^{2}} \frac{b^{2}}{\left(b^{2}+x_{3}^{2}\right)^{3 / 2}} \mathrm{~d} x_{3}-1\right) \\
& +\frac{\omega_{e}^{2}}{\omega^{2}} \frac{1}{N_{e}} \int_{0}^{\infty} \frac{\partial N_{e}}{\partial b} \mathrm{~d} x_{3}
\end{aligned}
$$

where the first term is the vacuum gravitational deflection; the second term is the additional deflection due to the presence of a homogeneous plasma; the third term is the deflection due to the plasma inhomogeneity (the refraction); $\omega$ is the photon frequency; and $\omega_{e}$ is the electron plasma frequency. The plasma frequency is determined by the density and mass of the ionized gas, i.e. $\omega_{e}^{2}=4 \pi e^{2} N_{e} / m_{e}$. The refractive index of the plasma is given by $n=1-\omega_{e}^{2} / \omega^{2}$, and thus the phase speed of light in the plasma is $v=c / n$. $N_{e}$ is the number density of electron, $b$ is the impact parameter, and $x_{3}$ is the coordinate along the line of sight. The formula (Eq.7) is valid only for $\omega>\omega_{e}$, since light waves with $\omega<\omega_{e}$ do not propagate in the plasma. In the limit of $\omega>>\omega_{e}$, this formula reduces to the vacuum case. The presence of plasma changes the deflection angle with the difference from the vacuum case being strongest for long wavelengths, as $\omega$ approaches $\omega_{e}$. The homogeneous plasma (second term in Eq. 7) increases the deflection angle of gravitational lensing. The density of plasma in galaxies usually decreases with radius, thus the refraction deflection (third term in Eq. 7 is opposite to the gravitational deflection, since the refractive index of plasma is small than 1.

In general, the photon frequency is much larger than $\omega_{e}$. Only in the case of radio wavebands and high density plasma, does the difference from the vacuum case reach a few percent. In this paper, we study lensing with low frequency radio observations in order to assess the observational signature of plasma. Two plasma distribution models, typical of spiral and elliptical lensing galaxies are adopted in this work. We discuss them in turn.

In model 1, appropriate to a spiral lensing galaxy, we assume that the number density of electrons is $10 \mathrm{~cm}^{-3}$ (Gutiérrez \& Beckman 2010), which corresponds to a plasma frequency of $\sim 1.8 \times 10^{5} \mathrm{Rad} / \mathrm{s}$. The distribution of plasma around a real lens is complex, so we use a circular symmetric form as an approximation. This is consistent with observations from a nearby galaxy, M51 Gutiérrez \& Beckman 2010). Taking

$$
N_{e}(r)=N_{0} \mathrm{e}^{-r / r_{0}}
$$

where $N_{0}$ is the central density of plasma, $r$ is the radial distance from the centre of the galaxy, and $r_{0}$ is the scale radius of the central region. For $M 51, N_{0}=10 \pm 1 \mathrm{~cm}^{-3}$, and $r_{0}=10 \pm 1 \mathrm{kpc}$. The deflection can thus be simplified to

$$
\boldsymbol{\alpha}_{p l}=\boldsymbol{\alpha}\left(1+\frac{\omega_{e}^{2}}{2 \omega^{2}}\right)-\frac{\omega_{e}^{2}}{\omega^{2}} \frac{b}{r_{0}} F(b) \hat{\theta},
$$

where $\hat{\theta}$ is the unit vector of coordinates, and $F(b)$ is an integral given in the Appendix.

In general, the observational frequency is several orders of magnitude higher than the plasma frequency $\left(\omega_{e}^{2} / \omega^{2}<\right.$ $10^{-6}$ ). It is only possible to identify the plasma lensing effect in radio observations at low frequencies (e.g. a few hundreds $\mathrm{MHz}$ ). From algebra, one can see that the additional deflection due to the homogeneous plasma is much smaller than that due to the gradient of the plasma. Thus we only consider the additional deflection due to an inhomogeneous plasma in this paper, and use a simplified lens equation with plasma

$$
\boldsymbol{\alpha}_{p l}(\boldsymbol{\theta})=\boldsymbol{\alpha}(\boldsymbol{\theta})-0.031 \boldsymbol{\theta} f(\theta)
$$

where $\boldsymbol{\theta}$ is the angular coordinates vector from the centre of the galaxy. More detail about the derivation of Eq. 10 and 


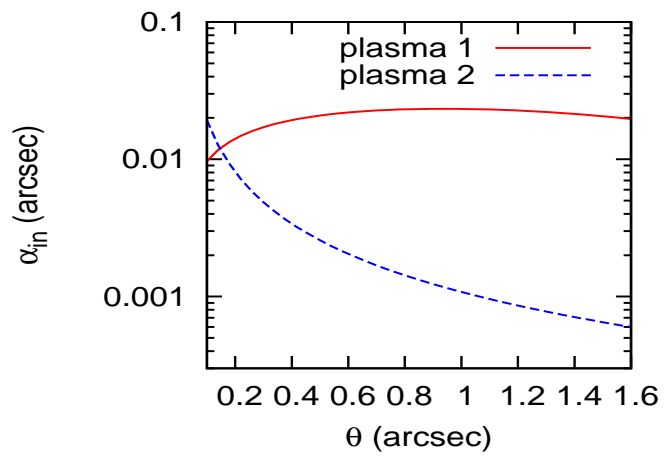

Figure 1. The extra deflection due to the inhomogeneous distribution of plasma. The solid red line represents the deflection angle of the first plasma model (Eq. 10, while the blue dashed line represents the second model (Eq. 12.

the integral function $f(\theta)$ can be found in Appendix A. Note that Eq. [10] is only valid for our model of plasma distribution and our observational frequency $(375 \mathrm{MHz})$. For general cases, Eq. 9. must be used instead.

In the second model of plasma in a lens galaxy, we use a more conservative estimate of the electron number density appropriate for an elliptical galaxy. It is given by Mathews \& Brighenti 2003)

$$
N_{e}(r)=N_{0}\left(r / r_{0}\right)^{-1.25}
$$

where $N_{0}=0.1 \mathrm{~cm}^{-3}$ is again the central density of plasma, and $r_{0}=10 \mathrm{kpc}$ is the scale radius. We also have a simplified lens equation for the second model

$$
\boldsymbol{\alpha}_{p l}(\boldsymbol{\theta})=\boldsymbol{\alpha}(\boldsymbol{\theta})-0.0006\left(\frac{\theta_{0}}{\theta}\right)^{5 / 4} \hat{\theta}
$$

where $\theta_{0}=1.6$ arcsec is the projected scale radius, and $\hat{\theta}$ is the unit vector. More detail is contained in Appendix B. In Fig 1 we compare the extra deflection angle due to two plasma models. As one might expect, the deflection due to the first plasma model is larger than that of the second model. The main reason is that the electron density in model 2 is smaller than that in model 1. Both kinds of galaxies (spiral and elliptical) have been found as lens galaxies in real observations. The plasma distributions that we use here are two typical models for electron density: the spiral galaxy is an upper limit, while the elliptical galaxy is a lower limit.

Throughout this paper, we adopt the same mock lens galaxy with both plasma models. The lens redshift is 0.5 , and the source redshift is 2.0. We use a Singular Isothermal Ellipsoid (SIE) for the lens mass model with a velocity dispersion of $\sigma_{v}=140 \mathrm{~km} / \mathrm{s}$ (which corresponds to $\theta_{\mathrm{E}}=0.40 \operatorname{arcsec}$ at this redshift), and ellipticity $\epsilon=0.3$.

\section{EFFECT ON SHEAR}

The additional deflection angle due to plasma decreases with increasing angular separation $\theta$. The deflections are generally small and can be neglected with large $\theta$, e.g. $\theta>10$ arcsec. Moreover, the plasma models are no longer valid at large radius. We thus only consider small angular separations in this paper.

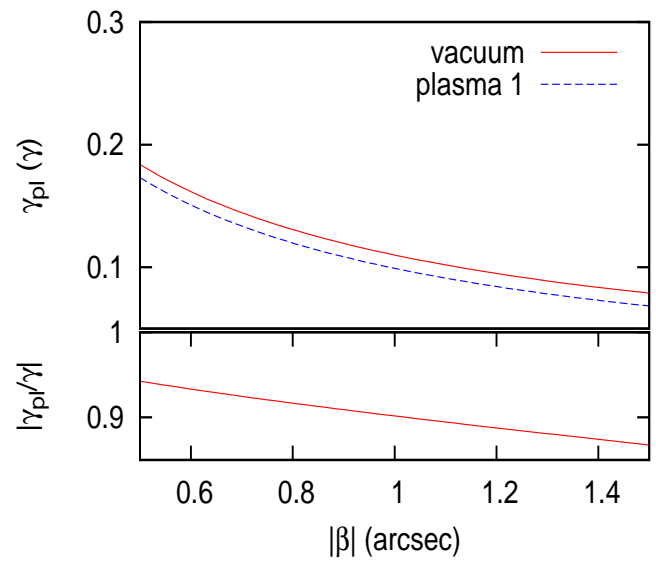

Figure 2. Estimates of weak lensing shear using ellipticity for the plasma model 1. In the top panel, the solid (dashed) line represents the estimated shear as a function of the source position in vacuum (plasma) lensing. In the bottom panel, the line shows the ratio of estimated shear of the plasma lensing over vacuum lensing as a function of source position.

We perform numerical simulations to test the changes in shear due to plasma. A circular-symmetric light profile is used for the source image

$$
I(\beta)=I_{e} \exp \left[-7.67\left(\left(\beta / \beta_{e}\right)^{1 / 4}-1\right)\right],
$$

where $I_{e}=1$ in arbitrary units, and $\beta_{e}=0.05$ arcsec. We move the source along the $\beta_{2}$ direction from $(0.2,0)$ to $(0.2,2)$ in order to generate a series of images. The lens equations in vacuum and with plasma (Eq.10) are used separately to generate two sets of lensed images. We use the second order brightness moments as an estimate of the ellipticity (shear) (Kaiser et al. 1995)

$$
\gamma=\frac{Q_{2}}{2 Q_{0}}=\frac{\int \mathrm{d}^{2} \theta I(\theta) \theta^{2}}{2 \int \mathrm{d}^{2} \theta I(\theta) \theta \theta^{*}} .
$$

We compare the estimated ellipticity between the two sets of images (Figs. 2] and 3). We can see that the presence of plasma increases the ellipticity of lensed images. In the case of very low frequency band observations and the high density plasma model 1, the estimated deviation can reach $\sim 5-$ $10 \%$. Although the fractional changes become slightly larger at large radius, the shear decreases more rapidly. The effect of plasma at large radius is in fact more difficult to observe. In the plasma model 2 , there is only about $\sim 1 \%$ difference.

Moreover, as we will see in the next section, the image positions are also slightly changed due to plasma. The combined effects (shear increasing and position shift) will change the estimated shear power spectrum, and cause slight systematics in the study of cosmic shear.

\section{EFFECTS ON STRONG LENSING}

\subsection{Image positions and magnifications}

Lensing magnification in presence of plasma is more complicated than shear since it is not linearly related to the 


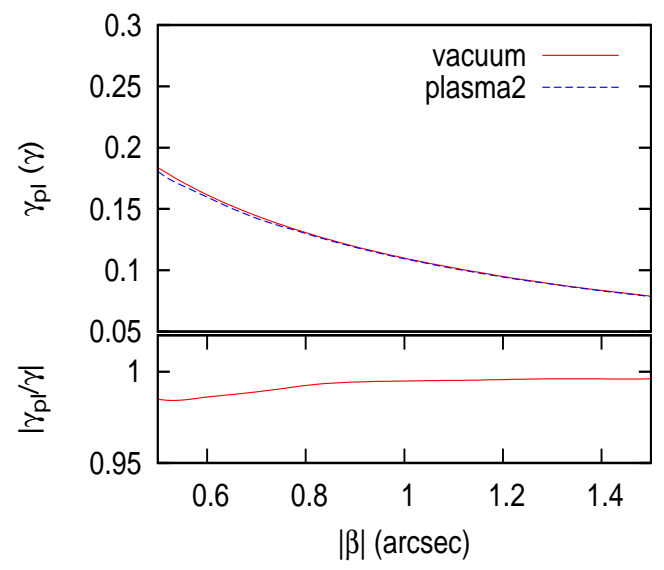

Figure 3. Same as Fig. 2 but for plasma model 2.

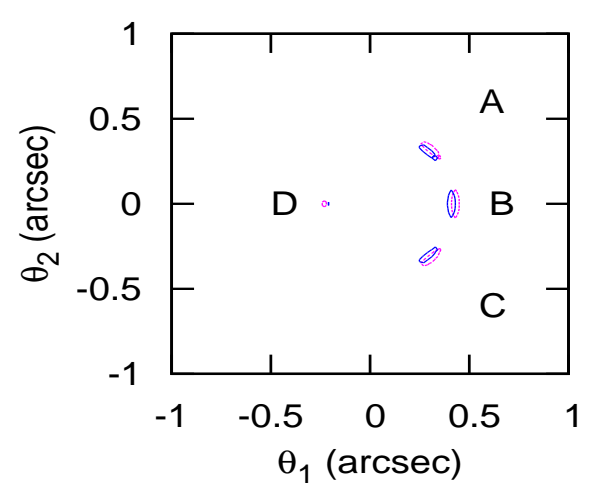

Figure 4. Two strong lensing multiple images: the purple images are the ones generated by vacuum lens equation, while the blue images are those with plasma (model 1). The lens is at the origin of the figure. The images are labelled from A to D.

deflection angle. Moreover, the absorption and scintillation of plasma will change the luminosity of the images, and the precise effect is wavelength dependent (see e.g. Scalo \& Elmeareen 2004). We will not consider the absorption effect since it is not significant in radio observations. The magnifications of the multiple images are changed differently by plasma.

With the same lensing properties as in the previous section, we create a mock multiple image strong lensing system. The source is placed at $(0.1,0.0)$ with a similar bright profile as in Eq. 13). We use a more compact source $\left(\beta_{e}=0.01\right.$ arcsec) in order to isolate the magnification effect. In Fig. 4 we can see that four images (labelled $A$ to $D$ ) are generated by lensing. Similar strong lensing systems have been found in the Universe (e.g. Bradač et al. 2002). The magnification $\mu$ and magnification ratio of the multiple images are given in Table 1. The images generated by plasma lensing (blue in Fig. 4 have slight smaller image separations. We can fit the plasma lensing images with the vacuum lens equation.
The strong lens modelling is complicated and beyond the scope of this paper, and one can find more detail from e.g. Suyu et al. (2009) and Veqetti \& Koopmans (2009). We evaluate only two parameters, the source position and the Einstein radius, and assume accurate knowledge of the other parameters. We obtain a slightly different source position and Einstein radius, $\beta=(0.095,0), \theta_{\mathrm{E}}=0.382$ arcsec, which is about $5 \%$ smaller than the input value. For the plasma model 2 , we obtain $\beta=(0.099,0), \theta_{\mathrm{E}}=0.401$ arcsec, which is only $1 \%$ different from the true model. The magnifications predicted by the models (also given in Table 1 ) have not significantly changed. The magnification cannot be observed directly since we do not know the intrinsic flux of the source. The flux ratio of multiple images can be directly compared between observations and model predictions. However, the magnification ratios are strongly affected by the lens properties, such as substructures Mao \& Schneider 1998; Xu et al. 2012). The effects due to plasma may be not as great as those due to substructures, and thus plasma has no important role in explaining the flux ratio anomalies.

\subsection{Time delay}

For a given source position, the excess time-delay surface as a function of position in the image plane is given by

$$
\begin{aligned}
t(\boldsymbol{\theta}) & =\frac{D_{d} D_{s}}{D_{d s}} \frac{1+z_{d}}{c}\left[\frac{1}{2}|\boldsymbol{\theta}-\boldsymbol{\beta}|^{2}-\psi(\boldsymbol{\theta})\right] \\
& \propto \frac{1}{H_{0}}\left[\frac{1}{2}|\boldsymbol{\theta}-\boldsymbol{\beta}|^{2}-\psi(\boldsymbol{\theta})\right]
\end{aligned}
$$

In the presence of plasma, the image position, $\theta_{p l}$, will be different. In reality, an extra effect should also come into the geometrical term $\frac{1}{2}|\boldsymbol{\theta}-\boldsymbol{\beta}|^{2}$ since the speed of light is different from $c$ in plasma. However, the refractive index is very close to 1 , even in the case of high density interstellar media and low frequency observations. The effect on the geometrical term is below the order of $10^{-5}$, and cannot be determined by current observational techniques. It will not be considered further here.

We calculate the time delay between multiple images, e.g., $\Delta t_{A B}=\left|t\left(\theta_{A}\right)-t\left(\theta_{B}\right)\right|$. The results of using vacuum and plasma lensing equations are given in Table 2. The difference in the time delay is about $0.2-1 \%$. The bottom line in Table 2 shows the time delay results calculated from fitting the model in the previous section. One can see that the difference is slightly larger.

By using the image configuration, one can model the mass distribution of the lens to determine the lens potential $\psi(\theta)$ and the unlensed source position $\boldsymbol{\beta}$. Lens systems with time delays can thus be used to study the cosmological parameters, e.g. Hubble constant Refsdal 1964, one can also read more detail from the COSMOGRAIL project, e.g., Courbin et al. 2011; Tewes et al. 2013). One can also see that there is a degeneracy between the cosmology and the lens potential, in that the mass-sheet degeneracy will cause an underestimate of the Hubble constant (e.g. Schneider \& Sluse 2013). We use the mock data generated from plasma lensing and use the vacuum lensing equation to reconstruct the mass distribution, and then calculate the Hubble constant. Other cosmological parameters, such as 


\begin{tabular}{ccccccccccc}
\hline \hline & $\theta_{A}$ & $\theta_{B}$ & $\theta_{C}$ & $\theta_{D}$ & $\mu_{A}$ & $\mu_{B}$ & $\mu_{C}$ & $\mu_{D}$ & $\mu_{A} / \mu_{B}$ & $\mu_{A} / \mu_{D}$ \\
\hline vacuum & $(0.304,0.322)$ & $(0.432,0)$ & $(0.304,-0.322)$ & $(-0.23,0)$ & 4.39 & 5.13 & 4.39 & 0.811 & 0.856 & 6.33 \\
\hline plasma 1 & $(0.290,0.310)$ & $(0.413,0)$ & $(0.290,-0.310)$ & $(-0.217,0)$ & 4.13 & 4.83 & 4.13 & 0.734 & 0.855 & 6.57 \\
\hline fitting 1 & & & & & 4.37 & 5.09 & 4.37 & 0.816 & 0.860 & 6.23 \\
\hline plasma 2 & $(0.302,0.322)$ & $(0.429,0)$ & $(0.302,-0.322)$ & $(-0.225,0)$ & 4.40 & 5.15 & 4.40 & 0.821 & 0.854 & 6.28 \\
\hline fitting 2 & & & & & 4.33 & 4.98 & 4.33 & 0.823 & 0.868 & 6.05 \\
\hline \hline
\end{tabular}

Table 1. The first four columns are the positions of lensed images (in units of arcsec). The other columns are magnifications and magnification ratios of the strong lensing multiple images with/without plasma. The third and fifth lines show predictions of magnification from fitting the vacuum to plasma model (see section 4.1 for more details). The positions and labels (A-D) are shown in the Fig. 4

\begin{tabular}{cccc}
\hline \hline & $\Delta t_{A B}$ & $\Delta t_{A C}$ & $\Delta t_{A D}$ \\
\hline vacuum & 0.454 & 0 & 6.21 \\
\hline plasma 1 & 0.453 & 0 & 6.20 \\
\hline fitting 1 & 0.424 & 0 & 5.66 \\
\hline plasma 2 & 0.454 & 0 & 6.21 \\
\hline fitting 2 & 0.481 & 0 & 6.18 \\
\hline \hline
\end{tabular}

Table 2. Time delay in units of day between lensed multiple images in vacuum, two plasma lensings and two model predictions. The image positions and labels are shown in Fig.4

$\Omega_{\Lambda}$, are unchanged in order to isolate the effect of plasma lensing in determining Hubble constant. The lensing model is fitted to the multiple image positions, given in the previous section. The Hubble constant can be estimated from two time delays: $h=0.628$ from $\Delta t_{A B}$ and $h=0.613$ from $\Delta t_{A D}$. Both results give significant underestimates at $\sim 9 \%$. For plasma model 2, we obtain $h=0.633$ from $\Delta t_{A B}$ and $h=0.668$ from $\Delta t_{A D}$.

Some analyses use optical observations for image positions and radio observations for the time delay measurement (Fassnacht et al. 2002; Suyu et al. 2010). In this case, the image positions are not modified by the plasma. Thus the lensing mass model is unbiased, while the time delay is still changed by the plasma. Such an approach will however lead to a slight underestimate for the Hubble constant. Using our mock lensing data, we obtain $h=0.673$ from $\Delta t_{A B}$ and $h=0.672$ from $\Delta t_{A D}$. The approach can cause an underestimate of up to $1 \%$ in the Hubble constant. In the second plasma model, there is no significant bias in the estimates.

\section{POLARIZATION AND MAGNETIC FIELD}

When a light ray passes through a plasma in the presence of a magnetic field, the polarization vector rotates due to the magnetic field. The rotation angle of the plane of polarization is given by (Sereno 2004; Morozova et al. 2013)

$$
\phi=\frac{e^{3} \lambda^{2}}{2 \pi m_{e}^{2} c^{4}} \int_{L} B_{\|}(l) N_{e}(l) \mathrm{d} l
$$

where $\lambda$ is the wavelength of the radiation as seen by the absorber medium, $B_{\|}$is the line of sight component of the magnetic field, and the integral is over the path length through the intervening absorbers. $m_{e}$ is the mass of the electron, and $e$ is the elementary charge. In multi-wavelength observations of polarization, the different rotation angles are determined from the Faraday Rotation Measurement (RM)

$$
R M=\frac{\Delta \phi}{\lambda_{\text {obs }}^{2}}=\frac{e^{3}}{2 \pi m_{e}^{2} c^{4}} \int B_{\|}(l) N_{e}(l)\left(\frac{\lambda}{\lambda_{\text {obs }}}\right)^{2} \mathrm{~d} l,
$$

where $\Delta \phi=\phi(\lambda)-\phi(0)$. In principle, from the RM one can estimate the average magnetic field of the lens

$$
\left\langle B_{\|}\right\rangle=\frac{\int N_{e}(l) B_{\|}(l) \mathrm{d} l}{\int N_{e}(l) \mathrm{d} l},
$$

where the denominator is the electron column density.

In reality measurement of polarization is difficult. Some results with a few percent polarization have been carried out (Joshi et al. 2007; Battye et al. 2011). The contamination along the line of sight, mainly from the Milky Way, is large. In multiple image lensing systems, the different images propagate along different lines of sight with different magnetic fields and will experience different RMs. We can use the differences to estimate the variation of magnetic field around the lens (Kemball et al. 2001). This estimate is independent of contamination along the line of sight since the angular separation is small, and the variation on such scales can be neglected. The time delay effect will change the RM due to source variations. Thus one has to calibrate the RM at the same emission time. We do not attempt to model the polarization within this paper since there is not sufficient observation result.

\section{SUMMARY AND DISCUSSION}

In this paper, we have studied gravitational lensing in the presence of plasma in the lens galaxy. Usually the lens galaxy/cluster is surrounded by plasma, and an extra deflection angle is caused by the plasma, especially by plasma inhomogeneity. By adopting a plasma distribution model in the lens galaxy, we have obtained a plasma lensing equation for a given range and observational frequency. The presence of plasma can cause several changes in lensing: the positions and magnifications of the lensed images, shear, and the time delay between the multiple images in strong lensing are all altered. 
In general, as most lenses are elliptical galaxies, the electron number density in the galaxy is probably as low as the second model we used in this study. Thus the effect due to plasma in lensing may not be significant. However, galaxies at high redshift may be different with denser ionized gas. The inclusion of plasma lensing provides a way to estimate the density of plasma in the lens. The greater the difference between image positions in different wave bands, the higher the density of plasma.

The effects due to plasma are only significantly observable in very low frequency observations. Radio observations using VLBI can reach very low frequencies ( $100 \mathrm{MHz}$ ), and very high spatial resolution (milli-arcsec). The plasma frequency in a high electron density region can reach $\sim$ $10^{6} \mathrm{~Hz}$. Under such conditions, the signatures due to plasma in lensing can reach a few percent. Magnification ratios between multiple images are mainly determined by the overall mass distribution and any inhomogeneous mass distribution within the lens, i.e. substructures. Therefore, the effect due to plasma on magnification ratios will be hard to detect. On the other hand, the impact of plasma on image positions and time delays may be discernible. It will be possible to use the image position differences between radio and optical observations to estimate the plasma density. Moreover, the effects on the time delay will bias estimates of the Hubble constant determined using low radio frequency. One should take the plasma effect into account in estimating the Hubble constant from low frequency radio observations.

However, intrinsic source images are different at different wavelengths. Especially in radio observations, the source size is larger than that in the optical. This effect will cause differential magnifications (Er et al. 2013), and thus introduce more difficulty in the reconstruction of the lens and source. Nevertheless, the observations from different wavelengths provide more information on both the lens and source.

Due to difficulties in the measurement of polarization and the contamination along the line of sight, little knowledge of lens magnetic fields has been obtained. With the help of new radio telescopes, such as LOFAR and SKA, it may be possible, using a large lens sample, to place constraints on the magnetic fields of lens galaxies or clusters.

One potential difficulty is that the ionosphere of the Earth affects radio transmission. It introduces systematics into the image positions, which drift around the sky with time. The state of the ionospheric plasma is difficult to describe, since it is strongly dependent on the activity of the Sun. For instance, the local winter hemisphere is tipped away from the Sun, and thus the ionosphere has less influence. The typical electron column at night ${ }^{3}$ is $\sim 10^{17} \mathrm{~m}^{-2}$. The electron column density in the galaxy is larger $\sim 10^{25} \mathrm{~m}^{-2}$ (under the assumption of $n_{e}=0.1 \mathrm{~cm}^{-3}$ and the thickness of the plasma is $10 \mathrm{kpc}$ ). However, in order to obtain precise image positions, we have to use long baseline interferometry. The states of the ionosphere at different telescope sites are different (Cotton \& Uson 2006). Calibration is essential but very difficult. Space radio telescopes, like Spekr-M4, may provide more information for calibration and observing lensing images. However, image stabilities at the milli-arcsec level are

\footnotetext{
3 http://iono.jpl.nasa.gov/latest_rti_global.html

${ }^{4}$ http://www.russianspaceweb.com/spektr_m.html
}

required by our study, but remain to be demonstrated in reality.

\section{ACKNOWLEDGMENTS}

We thank the referee for important comments, especially for pointing out a major mistake in the draft. We thank Neal Jackson, Richard J. Long, Lijun Gou and Olaf Wucknitz for useful comments on the draft. X.E. is supported by NSFC grant No.11203029. S.M. is supported by the Chinese Academy of Sciences and the National Astronomical Observatories of China.

\section{APPENDIX A: DEFLECTION ANGLE INTEGRAL OF MODEL 1}

In this section, we will neglect the effect of homogeneous plasma. The deflection angle caused by the plasma gradient is given by

$$
\alpha_{i n}=\int_{0}^{\infty} \frac{1}{\omega^{2}} K_{e} \frac{\partial N_{e}}{\partial b} \mathrm{~d} x_{3}
$$

where $K_{e}=4 \pi e^{2} / m_{e}$. The electron density we adopt in this paper is measured from the nearby galaxy M51 Gutiérrez \& Beckman 2010)

$$
N_{e}=N_{0} \mathrm{e}^{-r / r_{0}},
$$

where $N_{0}=10 \mathrm{~cm}^{-3}$ is a central value of electron density and $r_{0}$ is a scale length, which takes the value of $10 \mathrm{kpc} . r$ is the spherical radius $r=\sqrt{x_{3}^{2}+b^{2}}$. The impact parameter $b$ is approximately $b=\theta D_{d}$. The deflection angle can be written as

$\boldsymbol{\alpha}_{i n}(\boldsymbol{\theta})=-\frac{\omega_{0}^{2}}{\omega^{2}} \frac{\boldsymbol{\theta}}{\theta_{0}} F(\theta) \quad$ with $\quad F(\theta)=\int_{0}^{\infty} \frac{\mathrm{e}^{-\theta / \theta_{0} \sqrt{1+x^{2}}}}{\sqrt{1+x^{2}}} \mathrm{~d} x$,

where $\omega_{0}^{2}=K_{e} N_{0}$, and $\theta_{0}=r_{0} / D_{d}(\approx 1.6$ arcsec in our case). The integral function $F(\theta)$ can be approximated by a fitting function

$$
f(\theta)=3.6 \theta^{-0.22}-2.85,
$$

where $\theta$ is in units of arcsec and $f(\theta)$ is dimensionless. One should note that Eq. A4 is only valid for the plasma distribution used in this work, i.e. $\theta_{0}=1.6$ arcsec and is in the range of $[0.3,1.5]$ arcsec. Further, we assume a radio frequency $(375 \mathrm{MHz})$. The total deflection angle by gravity and plasma is calculated by

$$
\boldsymbol{\alpha}_{p l}=\boldsymbol{\alpha}-0.031 \boldsymbol{\theta} f(\theta)
$$

where the last term is given in units of arcsec.

\section{APPENDIX B: DEFLECTION ANGLE OF MODEL 2}

In the second plasma model, the plasma density is given by Mathews \& Brighenti (2003) and is appropriate for a typical elliptical galaxy

$$
N_{e}=N_{0}\left(r / r_{0}\right)^{-1.25}
$$




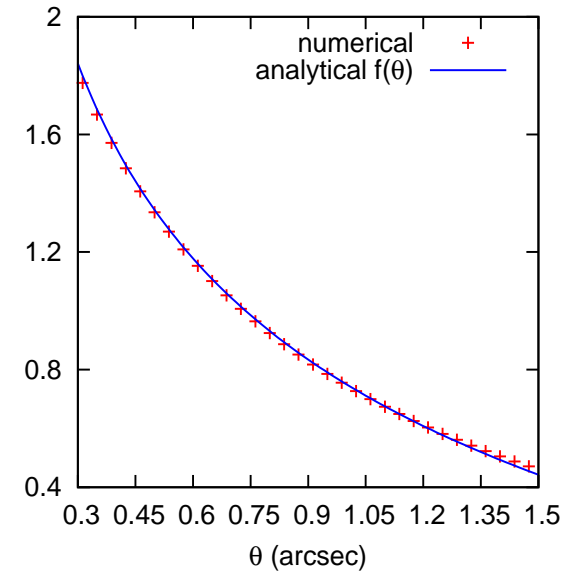

Figure A1. The integral function $F(\theta)$ (plus points) and its approximation $f(\theta)$ (solid line, Eq. A4.

where we have $N_{0}=0.1 \mathrm{~cm}^{-3}$ and $r_{0}=10 \mathrm{kpc}$. The deflection angle due to this inhomogeneous distribution of plasma is given by

$$
\alpha_{i n}=-\frac{\omega_{0}^{2}}{\omega^{2}}\left(\frac{r_{0}}{b}\right)^{5 / 4} \sqrt{\pi} \frac{\Gamma(9 / 8)}{\Gamma(5 / 8)} .
$$

For our lensing configuration and observation frequency (375 $\mathrm{MHz}$ ), the total deflection angle by gravity and plasma is calculated as

$$
\boldsymbol{\alpha}_{p l}=\boldsymbol{\alpha}-0.0006\left(\frac{\theta_{0}}{\theta}\right)^{1.25} \hat{\theta}
$$

where $\theta_{0}=1.6$ arcsec and $\hat{\theta}$ is the unit vector.

\section{REFERENCES}

Bartelmann, M. \& Schneider, P. 2001, Phys. Rep., 340, 291

Battye, R. A., Browne, I. W. A., Peel, M. W., Jackson, N. J., \& Dickinson, C. 2011, MNRAS, 413, 132

Bisnovatyi-Kogan, G. S. \& Tsupko, O. Y. 2009, Gravitation and Cosmology, 15, 20

Bisnovatyi-Kogan, G. S. \& Tsupko, O. Y. 2010, MNRAS, 404, 1790

Bradač, M., Schneider, P., Steinmetz, M., et al. 2002, A\&A, 388, 373

Cotton, Jr., W. D. \& Uson, J. 2006, in Bulletin of the American Astronomical Society, Vol. 38, American Astronomical Society Meeting Abstracts, 1015

Courbin, F., Chantry, V., Revaz, Y., et al. 2011, A\&A, 536, A53

Er, X., Ge, J., \& Mao, S. 2013, ApJ, 770, 110

Fassnacht, C. D., Xanthopoulos, E., Koopmans, L. V. E., \& Rusin, D. 2002, ApJ, 581, 823

Fu, L., Semboloni, E., Hoekstra, H., et al. 2008, A\&A, 479, 9

Gutiérrez, L. \& Beckman, J. E. 2010, ApJ, 710, L44

Joshi, S. A., Battye, R. A., Browne, I. W. A., et al. 2007, MNRAS, 380, 162

Kaiser, N., Squires, G., \& Broadhurst, T. 1995, ApJ, 449, 460

Keeton, C. R. \& Madau, P. 2001, ApJ, 549, L25
Kemball, A. J., Patnaik, A. R., \& Porcas, R. W. 2001, ApJ, 562,649

Koopmans, L. V. E., Biggs, A., Blandford, R. D., et al. 2003, ApJ, 595, 712

Li, G.-L., Mao, S., Jing, Y. P., Lin, W. P., \& Oguri, M. 2007, MNRAS, 378, 469

Li, R., Mo, H. J., Fan, Z., Yang, X., \& Bosch, F. C. v. d. 2013, MNRAS, 430, 3359

MacLeod, C. L., Jones, R., Agol, E., \& Kochanek, C. S. 2012, ArXiv: 1212.2166

Mao, S. \& Schneider, P. 1998, MNRAS, 295, 587

Mathews, W. G. \& Brighenti, F. 2003, ARA\&A, 41, 191

Morozova, V. S., Ahmedov, B. J., \& Tursunov, A. A. 2013, Ap\&SS, 346, 513

Narasimha, D. \& Chitre, S. M. 2008, ArXiv:0802.4044

Paraficz, D. \& Hjorth, J. 2010, ApJ, 712, 1378

Patel, P., Abdalla, F. B., Bacon, D. J., et al. 2013, ArXiv: 1303.4650

Patnaik, A. R., Menten, K. M., Porcas, R. W., \& Kemball, A. J. 2001, in Astronomical Society of the Pacific Conference Series, Vol. 237, Gravitational Lensing: Recent Progress and Future Go, ed. T. G. Brainerd \& C. S. Kochanek, 99

Planck Collaboration, Ade, P. A. R., Aghanim, N., et al. 2013, ArXiv: 1303.5076

Refsdal, S. 1964, MNRAS, 128, 307

Scalo, J. \& Elmegreen, B. G. 2004, ARA\&A, 42, 275

Schneider, P. \& Sluse, D. 2013, ArXiv: 1306.0901

Sereno, M. 2004, Phys.Rev.D, 69, 087501

Sluse, D., Hutsemékers, D., Lamy, H., Cabanac, R., \& Quintana, H. 2005, A\&A, 433, 757

Suyu, S. H., Marshall, P. J., Auger, M. W., et al. 2010, ApJ, 711, 201

Suyu, S. H., Marshall, P. J., Blandford, R. D., et al. 2009, ApJ, 691, 277

Suyu, S. H., Treu, T., Hilbert, S., et al. 2013, ArXiv: 1306.4732

Tewes, M., Courbin, F., Meylan, G., et al. 2013, A\&A, 556, A22

Treu, T. 2010, ARA\&A, 48, 87

Tsupko, O. Y. \& Bisnovatyi-Kogan, G. S. 2012, Gravitation and Cosmology, 18, 117

Tsupko, O. Y. \& Bisnovatyi-Kogan, G. S. 2013, Phys.Rev.D, 87, 124009

Vegetti, S. \& Koopmans, L. V. E. 2009, MNRAS, 392, 945

Xu, D. D., Mao, S., Cooper, A. P., et al. 2012, MNRAS, 421, 2553

York, T., Jackson, N., Browne, I. W. A., Wucknitz, O., \& Skelton, J. E. 2005, MNRAS, 357, 124 